\title{
In Silico Trials for Drug Safety and Efficacy Assessment Using a Novel Human Purkinje Fibre Model
}

\author{
C. Trovato ${ }^{1}$, C. Dusserre ${ }^{2}$, S. Billiald-Desquand ${ }^{2}$, E. Passini ${ }^{1}$, B. Rodriguez ${ }^{1}$ \\ ${ }^{1}$ Department of Computer Science, University of Oxford, Oxford, UK \\ ${ }^{2}$ Institut de Recherches Servier, Suresnes, FR
}

\begin{abstract}
In silico trials using multiscale and biophysicallydetailed human models have proven to be a new powerful tool for proarrhythmic risk prediction. Our goal is to evaluate the consistency between in silico results using a novel human Purkinje model and experimental data obtained from rabbit Purkinje fibres. The effects of Diltiazem, Dofetilide, Risperidone, and Verapamil were evaluated through AP biomarkers, at 1 and $0.2 \mathrm{~Hz}$. Druginduced effects on the ion channels were simulated through a simple pore-block model using IC50 values and Hill coefficients. Simulation results quantitatively reproduced the drug-induced effects experimentally observed for both Dofetilide and Risperidone. Simulations of Diltiazem and Verapamil correctly reproduced drug-induced effects observed experimentally in the early phase of repolarisation, but they showed AP prolongation instead of shortening. The interplay between $\mathrm{Ca}^{2+}$ and $\mathrm{K}^{+}$current inhibition was identified as the leading cause of the discrepancy. The experimentally observed AP shortening was quantitively matched when not considering the effects on $\mathrm{K}^{+}$current. In silico trials are a powerful tool for drug safety and efficacy assessment, and their performance requires careful evaluation of data describing drug-ionic current interactions since they critically affect the simulation results.
\end{abstract}

\section{Introduction}

Preclinical assessment of drug-induced safety and efficacy is still a challenge for pharmaceutical industries and regulators, which require new approaches [1]. In vitro, in vivo, and ex-vivo animal models are widely used for preclinical drug assessment, though they do not always translate to humans and represent a hefty cost for the pharmaceutical industry [2]. Cardiac Purkinje fibres, obtained often from dog or rabbit hearts, are one of the ICH S7B-recommended in vitro models for preclinical drug screening, especially for cardiotoxicity [3]. In silico trials using multiscale biophysically-detailed human-based models have proven to be a promising technology for cardiac safety prediction, achieving high level of accuracy for a large variety of compounds [4,5]. The productive integration of in silico trials for drug testing requires their evaluation against currently-used experiments. Our goal is to compare in silico simulations using a novel human Purkinje model with experimental data from rabbit Purkinje fibres and evaluate their consistency. We analysed the effects of four different compounds having different ionic profiles and different risk of inducing Torsade de Pointes (TdP) arrhythmia. We hypothesised that computational simulations could quantitatively predict drug-induced effects on cardiac Purkinje cells, and the model response needs to be interpreted considering the following elements: i) consistency of the $\mathrm{IC}_{50}$ values, especially for multi-channel blockers; ii) electrophysiological inter-species differences; iii) computational model characteristics, e.g. the balance of ionic currents and their dynamics to compensate the druginduced blocks.

\section{Methods}

\subsection{Experimental data}

The effects of Diltiazem, Dofetilide, Risperidone, and Verapamil were investigated in Purkinje fibres $(n=3)$ obtained from left ventricles of female rabbits $(\mathrm{N}=3)$ anaesthetised with sodium pentobarbital $(0.9$ to $1.0 \mathrm{~mL} / \mathrm{kg}$, intravenous) and maintained at $36 \pm 0.5^{\circ} \mathrm{C}$ in a $5 \mathrm{~mL}$-bath. Using the intracellular microelectrode technique, fibres were electrically stimulated at $1 \mathrm{~Hz}$ and superfused with Tyrode's solution, DMSO $0.1 \%$, and increasing concentrations of drugs, as reported in Table 1, for 30 minutes and washout with vehicle for 40 minutes. For each solution, after $1 \mathrm{~Hz}$, fibres were stimulated at $0.2 \mathrm{~Hz}$ for at least $3 \mathrm{~min}$ (to facilitate the occurrence of electrical abnormalities), followed by a 5-min recovery to $1 \mathrm{~Hz}$. The last ten action potential (AP) recordings for each concentration were used to compute the following biomarkers: resting membrane potential (RMP), AP durations at $40 \%, 50 \%, 70 \%$ and $90 \%$ of repolarisation $\left(\mathrm{APD}_{40}, \mathrm{APD}_{50}, \mathrm{APD}_{70}\right.$, and $\mathrm{APD}_{90}$ respectively), $\mathrm{AP}$ triangulation (APD90-40), AP amplitude (APA) and maximal rate of depolarisation $\left(\mathrm{dV} / \mathrm{dt}_{\max }\right)$. 
Based on the classification by CredibleMeds ${ }^{\circledR}$ [9], we considered Dofetilide and Risperidone as drugs with risk of inducing TdP, and Diltiazem and Verapamil as safe drugs (Table 1).

Table 1. Drugs, concentrations, TdP risk, and references to the $\mathrm{IC}_{50}$ values and Hill coefficients used in this study

\begin{tabular}{llcc}
\hline \multicolumn{1}{c}{ Drug } & $\begin{array}{c}\text { Concentrations } \\
(\mu \mathrm{mol} / \mathrm{L})\end{array}$ & $\begin{array}{c}\mathrm{TdP} \\
\text { risk }\end{array}$ & $\mathrm{IC}_{50}$ \\
\hline Diltiazem & $0.1 ; 1 ; 3 ; 10$ & No & {$[6,7]$} \\
Dofetilide & $0.001 ; 0.003 ;$ & Yes & {$[4,6,7]$} \\
& $0.01 ; 0.03$ & & \\
Risperidone & $0.1 ; 0.3 ; 1 ; 3$ & No & {$[6]$} \\
Verapamil & $0.1 ; 0.3 ; 1 ; 3$ & Yes & {$[7,8]$} \\
\hline TdP risk categories from CredibleMeds & {$[9]$}
\end{tabular}

\subsection{In silico drug trials}

In silico drug trials were performed for the same drugs and concentrations, using the Trovato2020 model, a recently published computational model of human cardiac Purkinje cellular electrophysiology [10].

Drug-induced ion current inhibition was simulated using a simple pore-block model, with $\mathrm{IC}_{50}$ and Hill coefficient from literature (Table 1) for: i) fast $\mathrm{Na}^{+}$current ( $\left.\mathrm{I}_{\mathrm{Na}}\right)$, ii) L-type $\mathrm{Ca}^{2+}$ current $\left(\mathrm{I}_{\mathrm{CaL}}\right)$, iii) rapid delayed $\mathrm{K}^{+}$ inward rectifier $\left(\mathrm{I}_{\mathrm{Kr}}\right)$. Extracellular concentrations were set as in the corresponding experiments. For Diltiazem, Dofetilide, and Verapamil more $\mathrm{IC}_{50}$ datasets were available as reported in Table 1. Simulations were repeated for each set of $\mathrm{IC}_{50}$ individually. Starting from steady state, the model was paced for 150 beats at either 1 or $0.2 \mathrm{~Hz}$. The same AP biomarkers considered for the experimental recordings were computed on the last beat.

All the simulations presented in this study were conducted using Virtual Assay [11] (v.3.2 (C) 2014 Oxford University Innovation Ltd. Oxford, UK), a user-friendly software package based on $\mathrm{C}++$, and specifically designed for in silico drug assays. Further analysis of the results was performed in Matlab (Mathworks Inc. Natwick, MA, USA).

\section{Results}

\subsection{Drug-induced effects at $1 \mathbf{H z}$}

Figure 1 shows simulated APs traces at $1 \mathrm{~Hz}$ for each of the drugs investigated in the present study. Dofetilide is a well-known Class III antiarrhythmic, selectively blocking IKr. In all the simulations (Figure 1A), Dofetilide induced concentration-dependent AP prolongation, with no relevant effects on RMP, APA, and dV/dtmax. The degree of AP prolongation varied depending on the $\mathrm{IC}_{50}$ values considered $[4,6,7]$, leading to an increase in the $\mathrm{APD}_{90}$ of
$81 \%, 40 \%$, and $105 \%$, respectively, at the maximum tested concentration $(0.03 \mu \mathrm{mol} / \mathrm{L})$. For all tested concentrations (Table 1), the first set of $\mathrm{IC}_{50}$ values led to AP prolongation consistent with the experimental observations in rabbit Purkinje fibre, whereas the second and third under and overestimated the magnitude of the AP prolongation, respectively.

Risperidone is an antipsychotic which is associated with QT prolongation and TdP arrhythmia. The simulated effects of Risperidone on the AP are reported in Figure 1B. Risperidone induced dose-dependent AP prolongations, with small effects on RMP, APA, and $\mathrm{dV} / \mathrm{dt}_{\text {max }}$. Simulations showed a good agreement with the experiments on the rabbit Purkinje in vitro model. At higher concentrations, 1 and $3 \mu \mathrm{mol} / \mathrm{L}$, the $\mathrm{APD}_{90}$ increased by $79 \%$ and $102 \%$ in line with the experimental observations. Decreasing the concentrations of one order of magnitude to 0.1 and $0.3 \mu \mathrm{mol} / \mathrm{L}$, led to a smaller APD 90 increase of $18 \%$ and $41 \%$, respectively, which overestimate the AP prolongations observed experimentally $(10 \%$ and $13 \%$, respectively). Finally, a small decrease $(<10 \%$ at the maximum concentration tested) in the $\mathrm{dV} / \mathrm{dt}_{\max }$ was observed in the experiments, which was not captured by the simulations.

Verapamil is a $\mathrm{Ca}^{2+}$ antagonist with different applications in the clinical setting, including treatment of arrhythmia. Two different experimental datasets were produced to investigate the effects of this drug. For the first dataset, rabbit Purkinje preparations were perfused for 7 minutes at each tested concentration (Table 1), whereas, preparations in the second dataset were perfused for 30 minutes. For both datasets, Verapamil showed mild effects on the RMP, APA and $d V / d t_{\max }$. However, in the first dataset, AP was shortened by Verapamil, whereas, in the second dataset AP was significantly prolonged. Simulations of Verapamilinduced effects are reported in Figure 1C for the two different sets of $\mathrm{IC}_{50}$ values $[7,8]$. In both cases, simulations yielded minor effects on the RMP and APA, and dose-dependent prolongation of the APs. At the maximum tested concentration $(3 \mu \mathrm{mol} / \mathrm{L})$, the $\mathrm{APD}_{90}$ increased by $51 \%$ and $59 \%$, consistently with the prolongation observed in the second experimental dataset. For all tested concentrations, simulations using the first $\mathrm{IC}_{50}$ set showed a better match to the experimental recordings than the second $\mathrm{IC}_{50}$ set.

In any case, simulations including the $\mathrm{IC}_{50}$ values for all the ionic currents were unable to reproduce the AP shortening observed in the first experimental dataset, due to a dominant role of $\mathrm{I}_{\mathrm{Kr}}$ compared to $\mathrm{I}_{\mathrm{CaL}}$ in setting the APD at the later repolarisation phase. In order to reproduce the AP shortening observed in the second set of experiments, additional simulations were performed for Verapamil, without including the effect on hERG. Figure $1 \mathrm{E}$ reports the simulated APs in for both $\mathrm{IC}_{50}$ sets $[7,8]$. 

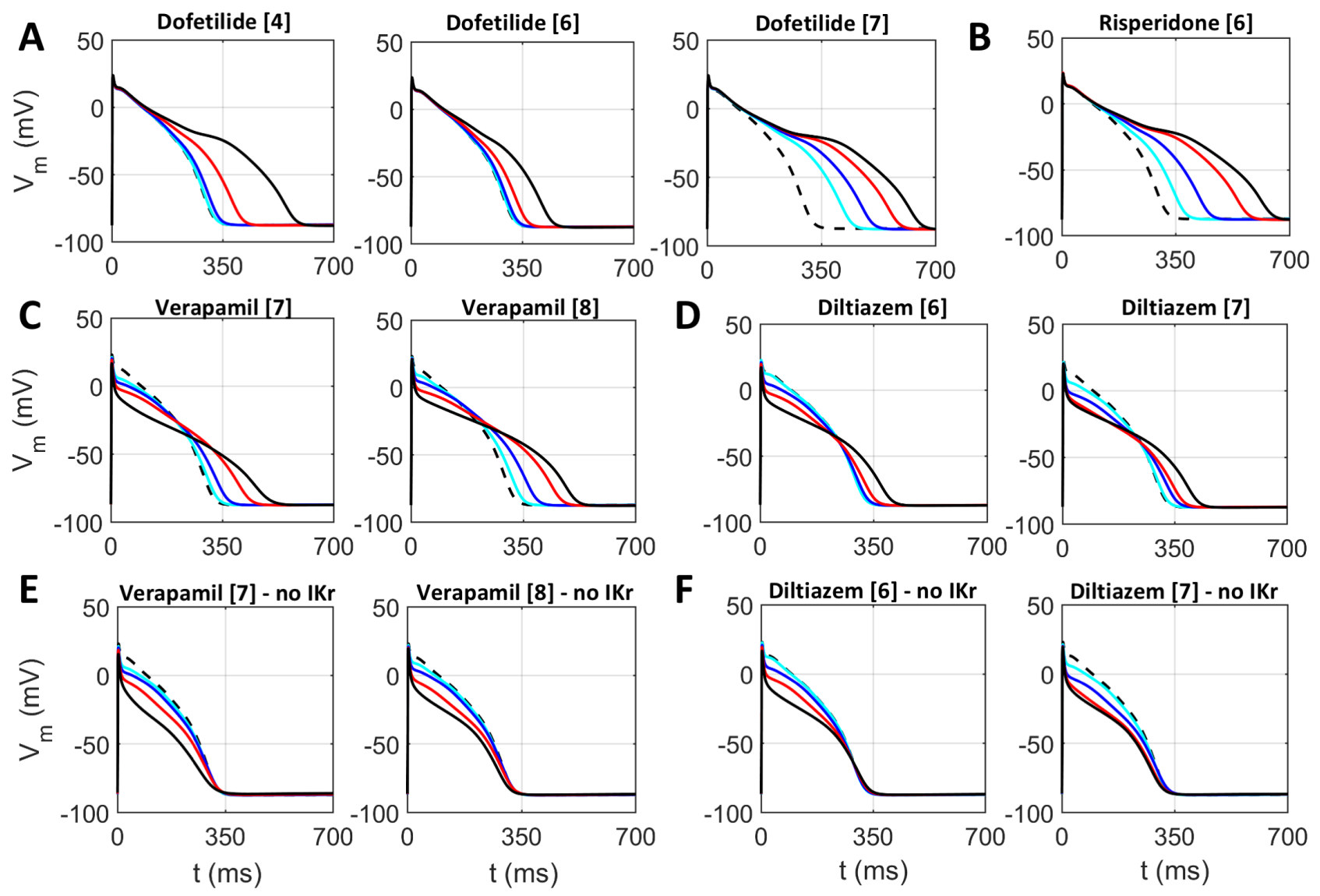

\begin{tabular}{|llllll}
\hline- Control Dose 1 & Dose 2 & Dose 3 & Dose 4 \\
\hline
\end{tabular}

Figure 1. Simulated AP for 12 in silico trials at $1 \mathrm{~Hz}$ for four different drugs. Each plot shows drug-induced effects on the $\mathrm{AP}$ at four concentrations of the same drugs and same $\mathrm{IC}_{50}$ and Hill coefficients, as reported in Table 1 and the plot title. A) Dofetilide; B) Risperidone; C) Verapamil; D) Diltiazem; E) Verapamil, without simulating of the inhibition of I $\mathrm{kr}$; F) Diltiazem, without simulation of the effects on IKr. The legend, common for all plots, is reported at the bottom of the figure and refers to the concentrations listed on in Table 1.

In this case, simulations showed AP shortening with a decrease in the $\mathrm{APD}_{90}$ of $7 \%$ and $5 \%$ at the maximum tested concentration, quantitively consistent with the second set of experiments.

Diltiazem is a Ca channel blocker, and it is used to treat hypertension and cardiac arrhythmias. Figure 1D shows simulated APs for Diltiazem, whose effects were represented using two different IC $_{50}$ datasets [6,7]. In both cases, simulations showed mild effects on RMP, APA, lowered AP plateau, and AP prolongations. The latter was not observed in the experiments.

Similarly to Verapamil, additional simulations were performed in order to match the AP shortening observed experimentally for Diltiazem, not including the $\mathrm{IC}_{50}$ for $\mathrm{IKr}_{\mathrm{Kr}}$ inhibition. In this case, simulations showed a better agreement with the experiments, leading to AP shortening for one set of $\mathrm{IC}_{50}$ [7], but not for the other [6].

\subsection{Drug-induced effects at $0.2 \mathrm{~Hz}$}

Figure 2 reports simulated APs at $0.2 \mathrm{~Hz}$ for Dofetilide, Risperidone, Verapamil, and Diltiazem. Both Dofetilide and Risperidone induced early afterdepolarisations at the higher concentrations tested (Table 1), in line with their known TdP risk and experiments in rabbits. Verapamil and Diltiazem induced AP prolongation an no repolarisation abnormalities for all the tested concentrations, consistently with their safety profiles. At slow pacing, both experiments with Verapamil showed AP prolongation, whereas, AP shortening was observed for Diltiazem.

\section{Discussion and Conclusion}

In this combined experimental and computational study, 

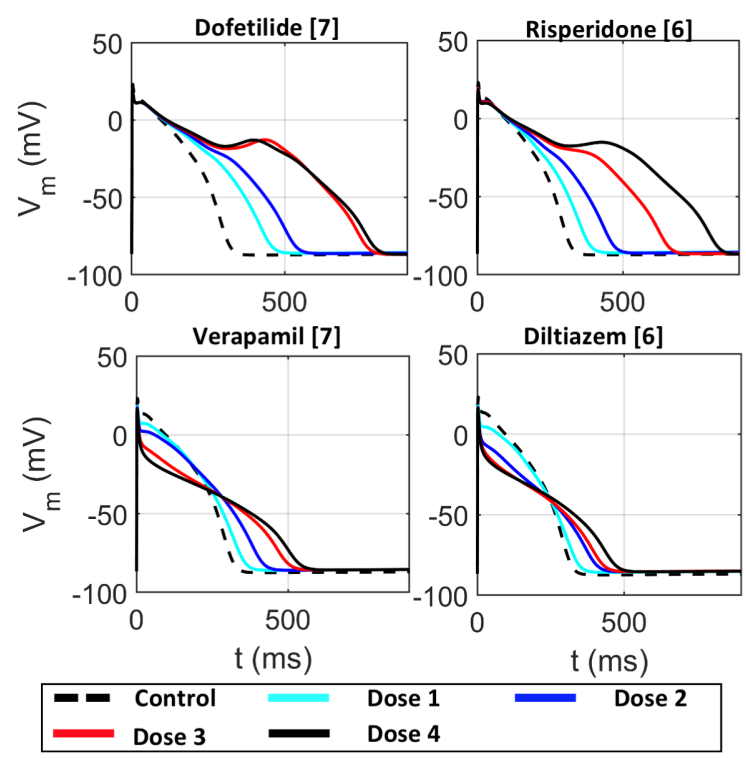

Figure 2. Simulated AP for in silico trials at $0.2 \mathrm{~Hz}$ for four different drugs. Each plot shows drug-induced effects on the AP at four concentrations of the same drugs and same $\mathrm{IC}_{50 \mathrm{~S}}$, as reported in Table 1 and in the plot title. The legend for all plots is reported at the bottom of the figure and refers to the concentrations listed in Table 1.

we compared the electrophysiological changes induced by 4 drugs (Diltiazem, Dofetilide, Risperidone, and Verapamil) on rabbit Purkinje fibre in vitro models and a human Purkinje in silico model.

Simulations of Dofetilide and Risperidone showed a quantitively good agreement between experimental and simulations, though in silico predictions were strongly dependent on the set of $\mathrm{IC}_{50}$ values chose to simulate the ionic current inhibitions. This was even more important for Verapamil and Diltiazem, due to the inhibition of both Ltype $\mathrm{Ca}^{2+}$ channel and hERG. In particular, experimental recordings with Verapamil showed two different behaviours, which could be reproduced by the model only including $\mathrm{I}_{\mathrm{CaL}}$ and $\mathrm{I}_{\mathrm{Kr}}$ inhibition, selectively. The main difference between the 2 sets of experiments was the drugexposure time, which suggest a different timing for the block of the L-type $\mathrm{Ca}^{2+}$ channel and hERG. Ultimately, $\mathrm{IC}_{50}$ values are recorded from heterologous expression systems and are static, capturing a specific time frame of the drug actions, and potentially missing more complex dynamic, especially when acting on multiple ion channels. Finally, species-differences between rabbit and human Purkinje fibres have been reported for the expression of the delayed rectifier $\mathrm{K}^{+}$channels subunits [12] though it is not yet clear how these differences affect the current dynamics, especially under drug action.

In silico drug trials are a powerful technology for drug safety assessment, and caution needs to be taken to the $\mathrm{IC}_{50}$ values that describe the drug behaviour since they critically affect the in silico model response.

\section{References}

[1] G.A. Van Norman, Limitations of Animal Studies for Predicting Toxicity in Clinical Trials: Part 2: Potential Alternatives to the Use of Animals in Preclinical Trials, JACC Basic to Transl. Sci. 5 (2020) 387-397.

G.A. Van Norman, Limitations of Animal Studies for Predicting Toxicity in Clinical Trials: Is it Time to Rethink Our Current Approach?, JACC Basic to Transl. Sci. 4 (2019) 845-854.

S. Goineau, M. Lemaire, G. Froget, Overview of safety pharmacology, Curr. Protoc. Pharmacol. 1 (2013) 1-8.

E. Passini, O.J. Britton, H.R. Lu, J. Rohrbacher, A.N. Hermans, D.J. Gallacher, R.J.H. Greig, A. BuenoOrovio, B. Rodriguez, Human In Silico Drug Trials Demonstrate Higher Accuracy than Animal Models in Predicting Clinical Pro-Arrhythmic Cardiotoxicity, Front. Physiol. 8 (2017) 1-15.

E. Passini, C. Trovato, P. Morissette, F. Sannajust, A. Bueno-Orovio, B. Rodriguez, Drug-induced shortening of the electromechanical window is an effective biomarker for in silico prediction of clinical risk of arrhythmias, Br. J. Pharmacol. 176 (2019) 3819-3833.

[6] J. Kramer, C.A. Obejero-Paz, G. Myatt, Y.A. Kuryshev, A. Bruening-Wright, J.S. Verducci, A.M. Brown, MICE Models: Superior to the HERG Model in Predicting Torsade de Pointes, Sci. Rep. 3 (2013) 2100.

[7] W.J. Crumb, J. Vicente, L. Johannesen, D.G. Strauss, An evaluation of 30 clinical drugs against the comprehensive in vitro proarrhythmia assay (CiPA) proposed ion channel panel, J. Pharmacol. Toxicol. Methods. 81 (2016) 251-262.

[8] C.A. Obejero-Paz, A. Bruening-Wright, J. Kramer, P. Hawryluk, M. Tatalovic, H.C. Dittrich, A.M. Brown, Quantitative profiling of the effects of vanoxerine on human cardiac ion channels and its application to cardiac risk, Sci. Rep. 5 (2015) 1-12.

[9] R. Woosley, K. Romer, www.Crediblemeds.org, QTdrugs List [Accessed 2020-04-29], AZCERT, Inc. 1822 Innov. Park Dr., Oro Val. AZ 85755. (1999).

[10] C. Trovato, E. Passini, N. Nagy, A. Varró, N. AbiGerges, S. Severi, B. Rodriguez, Human Purkinje in silico model enables mechanistic investigations into automaticity and pro-arrhythmic abnormalities, J. Mol. Cell. Cardiol. 142 (2020) 24-38.

[11] E. Passini, X. Zhou, C. Trovato, O.J. Britton, A. BuenoOrovio, B. Rodriguez, The virtual assay software for human in silico drug trials to augment drug cardiac testing, J. Comput. Sci. (2020) 101202.

[12] A. Atkinson, S. Inada, J. Li, J.O. Tellez, J. Yanni, R. Sleiman, E.A. Allah, R.H. Anderson, H. Zhang, M.R. Boyett, H. Dobrzynski, Anatomical and molecular mapping of the left and right ventricular His-Purkinje conduction networks, J. Mol. Cell. Cardiol. 51 (2011) 689-701. doi:10.1016/j.yjmcc.2011.05.020.

Address for correspondence:

Cristian Trovato

Dept. of Computer Science, 15 Parks road, Oxford, OX1 3QD. Cristian.trovato@gmail.com 